\title{
O discurso sobre álcool na Revista Brasileira de Enfermagem: 1932-2007
}

\author{
Gislene Farias de Oliveira ${ }^{1}$ \\ Luciana Barizon Luchesi²
}

Este é um estudo de revisão de literatura que analisou o tema álcool nos artigos da Revista Brasileira de Enfermagem, de 1932 a 2007. Realizou-se busca manual, utilizando as palavras-chave: álcool, alcoolismo, drogas e substâncias psicoativas. O discurso sobre álcool, utilizado pelos autores, foi analisado segundo o referencial metodológico de Bardin. Foram encontrados oito artigos que evidenciaram três categorias: aspectos biopsiquicossociais, concepções do álcool e do alcoolismo e imagem do alcoolista. O estudo demonstra a evolução no discurso sobre o álcool e alcoolismo de senso comum para a cientificidade, correspondendo ao momento histórico de sua produção.

Descritores: Alcoolismo; Características da Família; Atenção Primária à Saúde.

\footnotetext{
${ }^{1}$ Psicóloga, Professor Doutor, Faculdade de Medicina, Universidade Federal do Ceará, Brasil. E-mail: gislenefo@hotmail.com.

2 Enfermeira, Doutor em Enfermagem, Professor Doutor, Escola de Enfermagem de Ribeirão Preto, Universidade de São Paulo, Centro Colaborador de la OMS para el Desarrollo de la Investigación en Enfermería, SP, Brasil. E-mail: luchesi@eerp.usp.br.
}

Correspondencia:

Luciana Barizon Luchesi

Universidade de São Paulo. Escola de Enfermagem de Ribeirão Preto

Av. Bandeirantes, 3900

Bairro Monte Alegre

CEP: 14040-902 Ribeirão Preto, SP, Brasil

E-mail: luchesi@eerp.usp.br 


\title{
El discurso sobre el alcohol en la Revista Brasileña de Enfermería: 1932-2007
}

Se trata de un estudio de revisión de la literatura, que examinó el tema del alcohol en los artículos de la Revista Brasileña de Enfermería de 1932 a 2007. Se realizó una búsqueda manual a través de las palabras clave: alcohol, alcoholismo, drogas y sustancias psicoactivas. El discurso sobre el alcohol, usado por los autores, fue analizado de acuerdo a la metodología de Bardin. Fueron encontrados ocho artículos que mostraron tres categorías: los aspectos biopsicosociales, las concepciones del alcohol y del alcoholismo y, la imagen del alcohólico. El estudio muestra cómo el discurso sobre el alcohol y el alcoholismo evolucionan del sentido común al científico, lo que corresponde al momento histórico de su producción.

Descriptores: Alcoholismo; Composición Familiar, Atención Primaria de Salud.

\section{The discourse on alcohol in the Brazilian Journal of Nursing: 1932-2007}

\begin{abstract}
This was a literature review study which examined the theme of alcohol in the papers of the Brazilian Journal of Nursing, from 1932 to 2007. A manual search was performed using the keywords: alcohol, alcoholism, drugs and psychoactive substances. The discourse on alcohol, used by the authors, was analyzed according to the methodological proposals of Bardin. Eight papers were found that showed three categories: biopsychosocial aspects, conceptions of alcohol and alcoholism, and image of the alcoholic. The study shows how the discourse on alcohol and alcoholism has progressed from common sense to scientific status, corresponding to the historical moment of its production.
\end{abstract}

Descriptors: Alcoholism; Family Characteristics; Primary Health Care.

\section{Introdução}

O uso do álcool na sociedade atual possui conotação diferenciada em relação às outras drogas. Seu caráter lícito, de baixo custo e fácil acesso Ihe confere aceitação social ampla e propagada através da cultura religiosa, regional e rituais sociais, dificultando seu enfrentamento. A indústria do álcool reforça a propagação por meio de campanhas que estimulam o uso e dificulta a visibilidade social do álcool como problema de saúde que representa o maior índice de utilização mundial entre as substâncias psicoativas.

O uso de substâncias psicoativas data de milhares de anos e podem ser encontrados vestígios de sua utilização desde as civilizações antigas. Na atualidade, o cigarro, o álcool e outras substâncias psicoativas, além de uso medicinal, cumprem papel cultural, recreacional e religioso, funcionando como um "lubrificante social"(1).

As substâncias psicoativas apresentam, como principal mediador, o neurotransmissor dopamina que está associado à sensação de prazer "em vias específicas do sistema de recompensa e de motivação", que sofrem adaptações em contato com essas substâncias, de forma que, ao passar o efeito, o usuário pode apresentar sensação de desconforto importante ${ }^{(2)}$

Desde 1976, Edwards e Gross(3) já haviam identificado uma das principais enfermidades relacionadas ao álcool: a síndrome de dependência alcoólica, cujas características muito se assemelham àquelas gerais da dependência de substâncias psicoativas.

Segundo os autores, esse estado de dependência estava caracterizado pelo aumento da frequência e quantidade da ingestão da bebida, a ponto de causar constrangimentos sociais. Com a evolução, a tolerância se instala a ponto de ser necessário o aumento da quantidade para obtenção de efeitos semelhantes, passando o indivíduo a sofrer sensações desagradáveis de ordem física e psicológica quando não está sob o efeito da substância. Buscando minimizar ou evitar esse estado desagradável, o indivíduo passa a sentir pressão psicológica para fazer uso contínuo da substância, mantendo os níveis de álcool em patamar que lhe seja mais confortável. O uso passa a ser também para o alívio dos efeitos colaterais e não somente pelo prazer. Caso o 
indivíduo consiga entrar em um padrão de abstinência, se houver uma recaída, os níveis de dependência anterior serão reinstalados de acordo com o grau de dependência e níveis de consumo da bebida anteriores, podendo variar de meses a dias ${ }^{(3)}$.

De acordo com o relatório da Organização Mundial da Saúde ${ }^{(4)}$, o consumo global de álcool tem aumentado nas últimas décadas, principalmente nos países em desenvolvimento. No mundo, o álcool é responsável por 1,8 milhões de mortes, equivalente a $4 \%$ do peso global de doenças, sendo que as maiores incidências se encontram na América e Europa. A proporção de uso nas Américas e Europa é bem maior nos homens, onde o álcool varia de $8-18 \%$ do peso global das doenças e 2-4\% para mulheres.

O uso de substâncias psicoativas tem preocupado a sociedade brasileira nas últimas duas décadas em virtude do aumento de consumo e precocidade com que vem atingindo os jovens e crianças. Observa-se, ainda, aumento de problemas correlatos ao uso do álcool como aumento da criminalidade, acidentes de trânsito, abandono escolar, entre outros. Ainda existe grande estigma social em relação aos usuários, falta de políticas públicas e aumento da demanda por serviços na rede de saúde ${ }^{(5)}$.

Em estudo sobre consumo de drogas, em 107 cidades brasileiras, com população acima de 200.000 habitantes, envolvendo 8.589 pessoas de 12 a 65 anos de idade, das diversas classes sociais, autores encontraram em seus resultados que $68,7 \%$ dos sujeitos usou álcool alguma vez na vida. A estimativa de grau de dependência do álcool foi de $11,2 \%$, sendo maior nos homens $(17,1 \%)$ que nas mulheres $(5,7 \%)^{(6)}$.

Considerando-se que o álcool representa problema social, desde os povos antigos, parece haver falhas ao longo do processo histórico na contenção de seus danos. Algumas delas podem ser devidas à concepção sobre o "beber" em cada sociedade ao longo da história.

A Enfermagem lida com indivíduos em seu cotidiano que apresentam problemas relacionados ao álcool. Nesse sentido, investigar a concepção que a Enfermagem teve ao longo de sua trajetória de produção científica, no Brasil, permite traçar um esboço sobre a evolução do discurso científico da Enfermagem sobre o álcool, e como esse discurso contribuiu para o cuidado em saúde.

Sendo assim, a proposta do presente estudo foi analisar a trajetória do discurso sobre álcool na produção científica da Revista Brasileira de Enfermagem (REBEn), no período de 1932 a 2007.

\section{Método}

Trata-se de estudo de revisão de literatura, utilizando-se a estratégia de análise de conteúdo para crítica dos resultados. A pesquisa foi realizada através de busca manual nos números da REBEn, no período de 1932-2007, devido à falta de indexação desse periódico em seus primórdios. Para localização dos artigos sobre álcool, houve seleção inicial através dos títulos e resumos dos artigos que contivessem as palavras-chave: álcool, alcoolismo, substâncias psicoativas ou drogas, em seguida os dados foram confrontados com a Biblioteca Virtual em Saúde (BVS), que contém os dados da REBEn, a partir de 1955. Não foram localizados novos artigos durante a conferência eletrônica. Nesse caso, entendase como artigo todas os itens publicados na REBEn, de cunho de pesquisa ou não.

A escolha dessa revista se deu por se tratar do periódico de Enfermagem mais antigo do Brasil, editado pela Associação Brasileira de Enfermagem (ABEn), desde 1932. A Revista Brasileira de Enfermagem configura-se como rico instrumento de análise, visto seu pioneirismo, ainda na década de 1930, e representar a voz da Enfermagem científica isoladamente, por quase três décadas ${ }^{(7)}$. Nesse sentido, estudar o conteúdo dos artigos desse periódico permite vislumbrar recorte do discurso que teve impacto em muitas escolas de Enfermagem do país e, consequentemente, na formação de profissionais.

No que se refere à análise dos artigos selecionados, utilizou-se a Análise de Conteúdo Temática proposta por Bardin(8), cujo objetivo é compreender o sentido das comunicações e suas significações explícitas e/ ou ocultas. Essa técnica propõe as seguintes etapas operacionais: constituição do corpus, formado pelos artigos selecionados; leitura flutuante, seguida de leituras mais intensivas que permitem melhor compreensão dos dados obtidos e codificação e recortes, onde se procede à decomposição do corpus, agrupando o material em categorias e subcategorias. Optou-se, neste estudo, por selecionar frases, nos artigos que preenchessem os critérios de inclusão demarcados pelas palavras-chave. Durante a análise foram também consideradas as citações utilizadas nos textos, uma vez que denotam a escolha do autor para a fundamentação de seu discurso.

\section{Resultados e Discussão}

Foram localizados oito artigos respectivamente nos anos 1934(R1), 1938(R2), 1979(R3), 1986(R4), 
1996(R5), 2004(R6), 2006(R7) e 2007(R8), e o desmembramento das frases possibilitou a localização de 306 unidades de análise.

As frases selecionadas foram agrupadas em 3 categorias (1- aspectos biopsiquicossociais, 2concepções do álcool e do alcoolismo e 3-imagem do alcoolista). As categorias foram inspiradas no Banco de Cadastros da Rede de Pesquisas Sobre Drogas do Sistema Nacional de Políticas Sobre Drogas, apresentado no II Seminário de Pesquisa em Álcool e Drogas, em Ribeirão Preto, no dia 17 de novembro de 2008, durante a palestra intitulada "Rede de pesquisa sobre drogas"(9).

\section{Categoria 1 - Aspectos biopsiquicossociais}

Com relação aos aspectos biopsiquicossociais, sobressaíram-se 8 subcategorias, a saber: 1. efeitos adversos (com 3 subcategorias: fisiológicos, sociais e psicológicos); 2. diagnóstico/constatação; 3. cultural/ simbólico; 4. prevenção; 5. genética; 6. tratamento; 7. prognóstico e 8. comorbidade.

A categoria aspectos biopsiquicossociais concentrou a maioria das frases $(74,5 \%)$, sendo efeitos adversos a subcategoria com maior número de frases em relação ao total (29,1\%); seguida pelas subcategorias diagnóstico/ constatação $(21,6 \%)$, cultural/simbólico (6,9\%), prevenção $(5,6 \%)$, genética $(4,6 \%)$, tratamento $(3,6 \%)$, prognóstico $(2 \%)$ e comorbidade $(1,3 \%)$.

Subcategoria 1: efeitos adversos

Observou-se discurso voltado para as consequências do álcool em termos de efeitos e sintomas, decorrentes do uso dessa substância. Nesse caso, observou-se referência a três tipos de efeitos adversos, mostrados a seguir.

Fisiológicos: localizados em 4 artigos (1938, 1979, 1996 e 2006), demonstrados na seguinte frase: "O álcool absorvido, em natureza, atua brutalmente sobre o sistema nervoso, lesando-o e, por seu intermédio, altera o funcionamento das glândulas endócrinas, donde se seguirá o viciamento dos humores orgânicos do metabolismo, isto é, da nutrição geral dos fenômenos vitais, enfim" (R2) $)^{(10)}$.

Sociais: localizados em 4 artigos (1934, 2004, 2006 e 2007), evidenciados nas seguintes frases: "A mãe bebia até cair nas ruas de Porto, donde era transportada pela polícia" (R2)(10); "A análise dos artigos mostrou a importância de considerar as necessidades psicossociais e a história de vida da mulher, pois possibilita a identificação e a intervenção na situação de violência doméstica e uso de drogas na gestação" (R8)(11).
Psicológicos: localizados em 4 artigos (1934, 1938, 1996 e 2004) como, por exemplo: "O álcool traz ao indivíduo a corrupção da sensibilidade, o embotamento da inteligência, a extinção da vontade, tornando-o incapaz de viver na família ou na sociedade" (R1)(12).

A visão da Organização Mundial da Saúde é de que o álcool possui consequências para a saúde e o ambiente social do alcoolista através da intoxicação, dependência, dentre outros efeitos bioquímicos. A intoxicação tem sido relacionada a problemas como acidentes de carro violência doméstica, doenças crônicas e problemas de ordem social. Tem aumentado as evidências de que o volume de álcool consumido e os padrões de beber são impactantes para a saúde ${ }^{(4)}$.

\section{Subcategoria 2: diagnóstico/constatação}

Foi localizada em todos os artigos analisados. Essa subcategoria aponta para a constatação de situação ou diagnóstico já estabelecido. "Coisa profundamente lamentável é que o alcoolismo é factor importante na mortalidade infantil" (R2)(10); "(...) o número de mulheres, que faz consumo de bebidas alcoólicas, aumentou e, consequentemente, o número de gestantes" (R5) ${ }^{(13)}$

Subcategoria 3: cultural/simbólico

Foi identificada em cinco artigos (1934, 1938, 1996, 2004 e 2006). O conteúdo faz referência a práticas e/ou ações sociais que se associam a crenças, comportamentos, valores e regras morais de um grupo de pessoas ou de determinada comunidade, como pode ser observada nas frases: "Não amamenta a mulher ou o seu leite não satisfaz ao ente que cria: após as mamaduras chora o mesmo e esperneia: surge logo a vizinha, na sua prática de mulher multípara indica um ótimo galactogênico - a cerveja" (R1)(12); "Essa tendência para o alcoolismo talvez esteja relacionada aos modelos tradicionais de beber em grupos" $(\mathrm{R} 6)^{(14)}$.

O consumo do álcool é algo impregnado na cultura mundial. Ao longo da história, o uso e o consumo de álcool e das drogas, de maneira geral, têm sido encontrados em quase todas as civilizações. O álcool relaciona-se também à religião. A Bíblia Católica possui dezenas de referências ao vinho e às consequências de seu uso abusivo, como exemplo, em Isaías 28:7 "Mas também estes titubeiam sob efeito do vinho, alucinados pela bebida; sacerdotes e profetas cambaleiam na bebedeira. Estão afogados no vinho, desnorteados pela bebida, perturbados em sua visão, vacilando em seus juízos". Por outro lado, ao incorporar o vinho em um de seus sacramentos mais importantes, como o sangue de Jesus Cristo, imprime ao vinho conotação sagrada(15). 
A presença constante nas refeições, comemorações, no convívio social de modo geral, associado à história de tolerância milenar de seu uso e ao caráter lícito, certamente colaboram para sua disseminação e tolerância na sociedade.

Subcategoria 4: prevenção

Foi localizada em três artigos (1986, 1996 e 2004). Nesse caso, as frases denotaram ações preventivas ou necessidade das mesmas: "Ao retornar às escolas estudadas, foram ministradas aulas sobre os assuntos reivindicados" (referente a estudo com 1.976 jovens de ensino médio, em 10 escolas de uma cidade do interior do Estado de São Paulo, cujos temas mais solicitados para as aulas foram tóxico, álcool e tabagismo) $(\mathrm{R} 4)^{(16)}$; "A urgência de melhor divulgação do exame pré-natal (...), não só esclarecendo sobre os riscos do consumo de bebidas alcoólicas durante a gravidez" (R5) ${ }^{(13)}$.

Subcategoria 5: genética

Localizada em três artigos (1934, 1938 e 1996). Essa subcategoria remete à possibilidade de transmissão de características biológicas de uma geração para outra, como pode ser observado na frase. "Filhos de alcoolistas, degenerados de corpo e de espírito, seres hereditariamente talhados para o mesmo caminho, campo de ação da tuberculose, fatores adjuvantes da decadência física e moral de uma nação" (R1)(12); se a mãe elitista crônica der à luz a uma criança com síndrome fetal alcoólica e, mesmo assim, continuar consumindo bebida alcoólica, durante a próxima gravidez provavelmente terá um bebê com defeitos ainda mais graves $(. ..)(\mathrm{R} 5)^{(13)}$.

Esse levantamento observou que, na década de 1930, o discurso mostra-se em grande parte estereotipado negativamente em relação aos descendentes do alcoolista. Posteriormente, no artigo de 1996, o discurso contempla conotação em relação aos possíveis fatores genéticos de forma científica e ética.

\section{Subcategoria 6: tratamento}

Foi localizada em 2 artigos (1934 e 2004). Essa subcategoria envolveu prescrições, terapia, ou sugeriram algum tipo de tratamento, como pode ser observado na frase: "(...) o enfermeiro é um profissional indispensável para o tratamento e a busca da recuperação do alcoolista e suas atitudes podem influenciar definitivamente no relacionamento com o paciente alcoolista e, consequentemente, favorecer o tratamento" (R7)(17).
A baixa incidência dessa subcategoria mostra coerência com o contexto histórico-social de cada artigo. Desde 1911, com a adesão do Brasil ao Tratado de Haia, o país buscou adequar-se às normas internacionais para coibir a oferta e consumo de drogas no país. Entretanto, as políticas voltadas para a solução do problema tiveram, em sua maioria, conotação punitiva e policial, focada no controle das drogas ilícitas, cuja criminalização se dava através de diversas leis que previam prisão e permanência em sanatórios. Na década de 70, os hospitais psiquiátricos passam também a garantir a exclusão social desses indivíduos(18).

Em 1971, a medicina psiquiátrica influenciou nova visão a respeito do uso das drogas, mesmo que ainda restrito ao âmbito da justiça penal, como pode ser observado na Lei 5726. Em 1976, a Lei 6368 novamente transfere o manejo do uso de drogas para a medicina psiquiátrica e o termo "viciado" é substituído por "dependente de drogas". Essa Lei propõe a criação de estabelecimentos voltados para o cuidado e tratamento dos usuários de drogas, na rede pública, bem como o atendimento hospitalar e extra-hospitalar. Entretanto, esses primeiros serviços de saúde estavam voltados aos usuários de drogas ilícitas, deixando à margem o

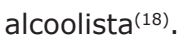

Em 2002, o Ministério da Saúde, influenciado pela III Conferência de Saúde Mental, lançou o Programa Nacional de Atenção Comunitária Integrada aos Usuários de Álcool e outras Drogas, sendo uma de suas estratégias a implementação dos Centros de Atenção Psicossocial para Álcool e Drogas (CAPSad), para municípios com mais de 200.000 habitantes, ou pertencentes às rotas de tráfico e fronteiras, sob a perspectiva da redução de danos, promovendo a volta do usuário como gestor de sua vida, sem exigir a abstinência compulsória, acolhendo o usuário e sua história dentro do sistema de saúde, sem julgamentos(19).

Subcategoria 7: prognóstico

Presença em dois artigos (1996 e 2004). O direcionamento do discurso foi no sentido de permitir ampliar um olhar para o futuro, a partir de um conhecimento já estabelecido: "É quase certo o fato de a tolerância levar à crescente necessidade, por parte do consumidor, de maiores quantidades de ingestão de etanol" (R5) (13).

\section{Subcategoria 8: comorbidade}

Encontrados dois artigos (1979 e 1996). O discurso foi utilizado para descrever a coexistência de doenças 
com o alcoolismo como observado na frase, referente à conclusão de estudo, realizado com 854 pacientes internados, em cidades do Estado de São Paulo: "Há associação entre alcoolismo e tuberculose pulmonar. No período menor que dois anos, que precede a doença, é mais forte a associação entre tuberculose pulmonar e bebedores excessivos" (R3)(20).

O uso abusivo do álcool, enquanto problema de saúde pública, deve ser enfrentado também em nível de atenção básica. Dessa forma, o Programa de Saúde da Família (PSF) apresenta-se como ferramenta de grande importância na identificação, prevenção e manejo das dificuldades relacionadas ao álcool e outras drogas. 0 vínculo estabelecido entre os profissionais, em âmbito familiar, pode potencializar a aceitação de relação de ajuda entre profissionais e família. Nesse aspecto, infere-se, aqui, ser o Programa de Saúde da Família ferramenta importante para a prevenção e tratamento de questões relativas ao álcool e ao alcoolismo, já que, através do programa, se tem acesso não somente aos pacientes, mas ao seu ambiente de convívio.

Por isso, faz-se necessária a capacitação de Enfermeiros, para a identificação e manejo de problemas relacionados ao álcool já no nível de atenção básica, bem como a facilitação do acesso do mesmo a informações, através de periódicos de Enfermagem e trabalhos científicos específicos atualizados, muitos dos quais disponibilizados na web, através da Biblioteca Virtual em Saúde (BVS), Periódicos Capes, Scientific Eletronic Library Online (SciELO), entre outros. Essa dinâmica é promotora de atuação mais eficaz frente ao alcoolismo e às drogas em geral.

\section{Categoria 2 - Concepções do álcool e do alcoolismo}

Essa categoria concentrou $17,3 \%$ das frases. Foram encontrados 4 artigos com referência a essa categoria nos anos 1934, 1938, 2004 e 2006 e se destacam duas subcategorias; uma desfavorável (16\%), que concentra a grande maioria das frases, onde os autores apresentam discursos que denotam as consequências negativas do álcool e do alcoolismo e do beber: "Álcool - flagelo da humanidade, deturpador dos tecidos na sua intimidade, dizimador das raças" $(R 1)^{(12)}$, e outra, de evidência social de tolerância, como apresenta a fala: "(...) o alcoolismo pode ser produtor de processos próprios de interação entre os trabalhadores, de tal modo que produza uma cultura alcoolista (...)" (R6)(14).
Algumas falas da década de 1930 instigam no leitor sentimentos de repulsa, coerente com o contexto nacional do período, onde ações governamentais se concentravam na coerção e até mesmo na punição, no caso do alcoolista vir a provocar "desordem social".

Existe uma cultura de beber para agradar, para fazer companhia, para receber e ser aceito em festas e em outras comemorações, sendo o vinho e outras bebidas alcoólicas entendidas como símbolo de comunicação social de alegria de viver. A cultura apresenta convites para a bebida, porém, o indivíduo que avança ao ponto do alcoolismo é rechaçado pela sociedade.

Além disso, as políticas públicas precisam lutar com a forte propaganda das empresas e indústrias de bebidas alcoólicas, que estimulam o uso da substância através de mensagens explícitas ou subliminares, quase sempre antagônicas às proposições educativas do governo.

\section{Categoria 3 - Imagem do alcoolista}

Com relação à categoria imagem do alcoolista, $8,2 \%$ das unidades de análise foram localizadas nesse tema, em 3 artigos (1934, 1938 e 2004). Os resultados destacam duas subcategorias: uma negativa (1), onde alguns autores apresentam uma série de referências pejorativas ao alcoolista, que desordenam e desgastam as condutas sociais, e a outra subcategoria - tolerância e necessidade de assistência de saúde (2), onde o alcoolista é visto como pessoa que precisa de ajuda e que deve ser tratado como um ser humano de forma holística, como pode ser observado, respectivamente, nas frases: 1) "Alcoólatra, tronco de uma geração degenerada, povoador de hospitais, hospícios e prisões, futuro hóspede de uma dessas instituições" $\left.(\mathrm{R} 1)^{(12)} ; 2\right)$ ", "Faz-se necessária a retirada do caráter de voluntariedade da ingestão de bebidas alcoólicas, abolindo o julgamento moralista em relação aos dependentes do álcool" $(R 7)^{(17)^{*}}$.

Nessa categoria, houve concentração maior de discursos alusivos à visão preconceituosa da imagem do alcoolista na década de 1930, coerente com a prática legal de envio de usuários às prisões e hospitais. Já em 2004, as falas negativas são voltadas para a descrição de crenças sociais, não se tratando de emissão de juízo de valor do autor.

O texto de 1934 (R1)(12) coloca a infância como ponto de avanço das nações e a mulher/mãe como principal responsável pela formação moral do filho,

* Nesse caso, deve-se destacar que o texto expressa a ideia do autor, mas, originalmente, se trata de citação de Ramos SP, Bertolote JM (org). Alcoolismo hoje. $3^{a}$ ed. Porto Alegre (RS): Artes Médicas; 1997. 
podendo mantê-lo longe do álcool e, ainda, mostrando que apenas a educação feminina formaria elementos importantes na luta contra o álcool. O texto de 1938 $(R 2)^{(10)}$ destaca a importância de se mudar a conotação social permissiva sobre a bebida alcoólica e se atentar para as propagandas que incentivam seu uso.

Os textos de 1986 (R4), 1996 (R5), 2004 (R6) e 2006 (R7) se referem à importância da realização de rastreamentos que podem ser desenvolvidos pela equipe de saúde, seja no contexto dos adolescentes (R4)(16), gestantes (R5)(13), no âmbito do trabalho $(R 6)^{(14)}$, ou entre os profissionais de enfermagem $(R 7)^{(17)}$.

Nesse sentido, os enfermeiros podem contribuir muito para o cuidado em saúde através de intervenções baseadas em inquéritos prévios, consolidados e validados cientificamente para entender o conceito que a população e os próprios profissionais possuem sobre a questão do alcoolismo, no sentido de aprimorar o atendimento e promover ações de saúde.

Como exemplo, para inquérito junto a profissionais da Enfermagem pode ser utilizada a Escala de Atitudes frente ao Álcool, ao Alcoolismo e ao Alcoolista (EAFAAA), desenvolvida recentemente em virtude de dificuldades sentidas por pesquisadores para o uso de escalas internacionais( ${ }^{(21)}$.

\section{Conclusão}

Os resultados deste estudo apontam para a representação do álcool enquanto droga que pode trazer inúmeras consequências orgânicas, familiares, profissionais e psicossociais. Houve ênfase no discurso dos artigos para os aspectos biopsiquicossociais em detrimento das questões de prevenção e tratamento.

De maneira geral, sugerem alguns pontos fundamentais. Os discursos dos profissionais de saúde, presentes na revista sobre o álcool, parece que recebem influência de seus contextos históricos/sociais e de políticas públicas. Dessa forma, as referências ao álcool evoluíram de pré-julgamentos e estereótipos negativos, além de estímulo à repreensão e desvalorização do alcoolista na década de 1930, para discurso voltado às políticas públicas de tratamento do dependente de álcool e um olhar ao alcoolismo enquanto problema de saúde pública e doença incapacitante, cujo manejo deve ser isento de julgamentos morais, com realização de estratégias de prevenção e redução de danos.

O aumento de estudos sobre o tema ampliará o espectro do conhecimento, bem como promoverá certamente maior visibilidade no meio científico, implicando em maiores discussões e reflexões sobre o álcool, suas consequências, formas de enfrentamento do problema, políticas públicas associadas e suas avaliações.

Deve-se considerar que o número reduzido de artigos encontrados foi surpreendente, uma vez que mais de 30 anos de REBEn foram pesquisados. Contudo, esse dado não pode ser generalizado para o contexto brasileiro, pois, ao longo das décadas, a criação de outros periódicos, inclusive alguns especializados na temática, como a Revista Eletrônica Saúde Mental Álcool e DrogasSMAD, pode ter diluído as publicações. Observa-se, atualmente, que as revistas indexadas na Biblioteca Virtual em Saúde (BVS), com maior número de artigos localizados, que indicam álcool como temática, são a Revista da Escola de Enfermagem Anna Nery e Revista Latino-Americana de Enfermagem. Entretanto, devese considerar que, entre os periódicos de enfermagem brasileiros, com exceção da Revista da Escola de Enfermagem da USP e Cogitare Enfermagem, as demais revistas foram criadas apenas na década de 1990, portanto, não permitiria análise histórica anterior.

A boa classificação e indexação em bases de dados importantes como BVS e SciELO ainda conferem à Revista Brasileira de Enfermagem status, no momento de escolha de pesquisadores para envio de seus trabalhos aos periódicos. Nesse sentido, a análise de seu discurso pode ser entendida como esboço inicial sobre o discurso científico da Enfermagem, de forma mais ampliada, podendo, em estudos futuros, avaliar se esse discurso encontrará reflexo nas demais revistas de Enfermagem criadas posteriormente.

Uma vez que um dos objetivos do trabalho dos profissionais de saúde, no âmbito da prevenção, é levar informações atualizadas e com base científica as pesquisas na área de Enfermagem terão grande impacto no discurso de seus profissionais.

\section{Agradecimentos}

Agradecemos a Comissão Interamericana para o Controle do Abuso de Drogas/CICAD da Secretaria de Segurança Multidimensional/SSM da Organização dos Estados Americanos/OEA, a Secretaria Nacional de Políticas sobre Drogas/SENAD do Gabinete de Segurança Institucional/Brasil, a Escola de Enfermagem de Ribeirão Preto da Universidade de São Paulo e o Centro Colaborador da Organização Mundial da Saúde para o Desenvolvimento da Pesquisa em Enfermagem, a população representada nas pesquisas, bem como as autoridades das universidades representadas pelos participantes do Programa On-Line de Especialização em Pesquisa sobre o Fenômeno das Drogas - PREINVEST, períodos 2005, 2006, 2007 e 2008. 


\section{Referencia}

1. Duhamel F. La santé et la famille: une approche systémique en soins infirmiers. Montréal:Gaëtan Morin Editeur, 1995.

2. US Department of Health and Human Services. Substance Abuse and Mental Health Services Administration. SA MASA. Results from the 2005 National Survey on Drug Use and Health. Rockville (MD): Office of Applied Studies: National Findings; 2006.

3. Grant BF. Estimates of US children exposed to alcohol abuse and dependence in the family. Am J Public Health 2000; 90(1):112-5.

4. National Institute on Alcohol Abuse and Alcoholism. Youth Drinking: Risk Factors and Consequences. Alcohol Alert [internet]. 1997; (37). Available from: http://pubs.niaaa.nih. gov/publications/aa37.htm

5. Hawkins JD, Graham JW, Maguin E. Exploring the effects of age of alcohol use initiation and psychosocial risk factors on subsequent alcohol misuse. J Stud Alcohol 1997; 58(3):280-90.

6. Andrews JA, Hops $H$, Ary D. Parental influence on early adolescent substance use: Specific and nonspecific effects. J Early Adolesc 1993; 13(3):285-310.

7. Ary DV, Tildesley $E$, Hops H. The influence of parent, sibling, and peer modeling and attitudes on adolescent use of alcohol. Int J Addictions 1993; 28(9):853-80.
8. Resnick MD, Bearman PS, Blum RW. Protecting adolescents from harm: Findings from the National Longitudinal Study on Adolescent Health. J Am Med Assoc 1997; 278(10):823-32.

9. McGue M, Sharma A, Benson P. Parent and sibling influences on adolescent alcohol use and misuse: Evidence from a U.S. adoption cohort. J Studies Alcohol 1996; 57(1):8-18.

10. Cesar C, Suarez A. Promociòn de la Salud y Prevenciòn de la Enfermedad, Enfoque en Salud Familiar. Cap 8. Colombia:Jàurigue Paulina; 2004.

11. Babor TF, Fuente JR, Saunders J, Grant M. Audit: The alcoholic use disorders identification test: Guidelines for use in primary health care. Geneva: World Health Organization; 1989. 12. Saunders JB, Aasland OG, Babor TF, Fuente JR, Grant M. Development of the Alcohol Use Disorders Identification Test (AUDIT):WHO collaborative project on early detection of persons with harmful alcohol consumption. II. Addiction 1993; 88:791804.

13. Harwood GA. Alcohol abuse - screening in primary care. Nurse Practitioner 2005; 30(2):56-61.

14. Ockene JK, Wheeler E, Adams A. Provider training for patient-centered alcohol counseling in a primary care setting. Arch Int Med 1997; 157(20):2334-41.

15. Vargas D, Luis MAV. Alcohol, alcoholism and alcohol addicts: conceptions and attitudes of nurses from district basic health centers. Rev. Latino-Am. Enfermagem 2008; 16(spe):543-50. 